\title{
HIGH FIELD MAGNETOTRANSPORT OF THE PRESSURE INDUCED ORGANIC SUPERCONDUCTOR (BEDT-TTF) ${ }_{3} \mathrm{Cl}_{2} 2 \mathrm{H}_{2} \mathrm{O}$
}

\author{
W. LUBCZYŃSKI
}

Department of Solid State Physics, Polish Academy of Sciences

Wandy 3, 41-800 Zabrze, Poland

J. Caulfield, J. Singleton, W. Hayes

Physics Department, The Clarendon Laboratory, University of Oxford Parks Road, Oxford 0X1 3PU, United Kingdom

M. KURMOO AND P. DAY

The Royal Institution, 21 Albemarle Street, London W1X 4BS, United Kingdom

The magnetotransport of single crystals of (BEDT-TTF) ${ }_{3} \mathrm{Cl}_{2} 2 \mathrm{H}_{2} \mathrm{O}$ was studied in the pressure range $10 \div 20 \mathrm{kbar}$ at continuous magnetic fields up to $15 \mathrm{~T}$ and at temperatures down to $700 \mathrm{mK}$. Above $12.5 \mathrm{kbar}$ a single series of Shubnikov-de Haas oscillations is observed, corresponding to about $0.5-1 \%$ of the cross-section of the first Brillouin zone. A superconducting state with the highest transition temperature close to $3 \mathrm{~K}$ is stabilised between $10.2 \mathrm{kbar}$ and 13.5 kbar.

PACS numbers: $72.15 . \mathrm{Nj}$

(BEDT-TTF) ${ }_{3} \mathrm{Cl}_{2} 2 \mathrm{H}_{2} \mathrm{O}$ is the only superconducting charge-transfer salt based on BEDT-TTF molecule where the BEDT-TTF ions each have charge $+(2 / 3) e$. The crystal structure of this compound is composed of the two-dimensional network of BEDT-TTF cations, separated by complex anions consisting of units of $4 \mathrm{Cl}^{-}$and $4 \mathrm{H}_{2} \mathrm{O}$ molecules. The calculated Fermi surface consists only of the one-dimensional sections [1] or, in another approach, of two parallel one-dimensional sections and an elongated closed electron pocket [2]. For both of these electron band structure models a little difference appears to be between liquid helium and room temperature.

(BEDT-TTF $)_{3} \mathrm{Cl}_{2} 2 \mathrm{H}_{2} \mathrm{O}$ is a semimetal under ambient pressure according to thermopower, susceptibility and conductivity measurements [3], but undergoes transition to an insulating state below about $100 \mathrm{~K}$. The application of pressure gradually reduced the transition temperature and stabilised a superconducting 
state below $4 \mathrm{~K}$, between $10 \mathrm{kbar}$ and $16 \mathrm{kbar}[4,5]$. To study the electronic band structure of (BEDT-TTF) ${ }_{3} \mathrm{Cl}_{2} 2 \mathrm{H}_{2} \mathrm{O}$ we undertook the pressure measurements of magnetotransport at high magnetic fields and low temperatures.

Standard 4-wire AC techniques $(5-150 \mathrm{~Hz}$ ) were employed for all measurements, with the current applied parallel to the high conductivity plane of the crystal platelet. Magnetoresistance measurements under hydrostatic pressure were performed using a nonmagnetic clamp cell filled with petroleum spirit. All pressures quoted are those measured at $4 \mathrm{~K}$.

Magnetoresistance of a single crystal of (BEDT-TTF) ${ }_{3} \mathrm{Cl}_{2} 2 \mathrm{H}_{2} \mathrm{O}$ is shown in Fig. 1. Evidence of the two-dimensionality of the free carriers is clearly observed as a single series of low frequency oscillations superimposed on the magnetoresistance, periodic in reciprocal field (Fig. 1, inserts). The Shubnikov-de Haas frequency

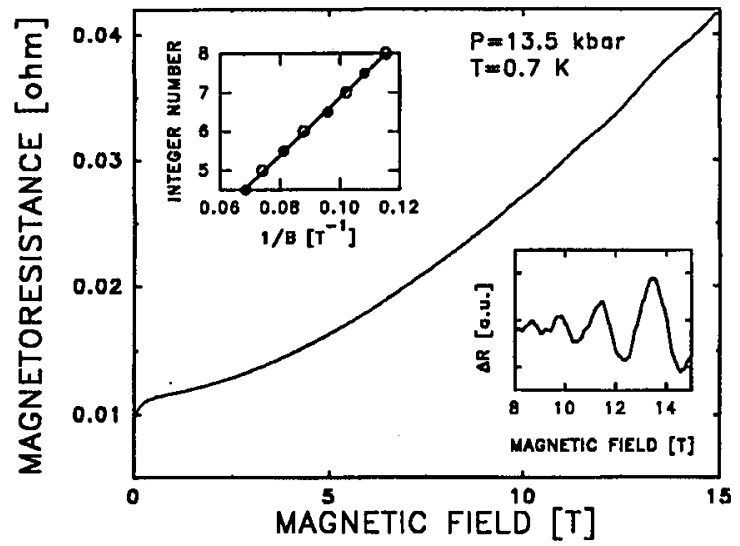

Fig. 1. The magnetoresistance of (BEDT-TTF) ${ }_{3} \mathrm{Cl}_{2} 2 \mathrm{H}_{2} \mathrm{O}$ crystal at $0.7 \mathrm{~K}$ under hydrostatic pressure of $13.5 \mathrm{kbar}$. The upper inset is a plot of the oscillation position (in reciprocal field) versus the Landau level index from the oscillatory part of the magnetoresistance presented in lower inset. The solid line has a gradient of $74 \mathrm{~T}$, filled dots correspond to magnetoresistance dips and open dots - to magnetoresistance peaks.

increases from $45 \mathrm{~T}$ at $12.5 \mathrm{kbar}$ and asymptotically approaches $100 \mathrm{~T}$ at $20 \mathrm{kbar}$ (Fig. 2). The mass of the carriers obtained from fitting the temperature dependence of the amplitude of the Fourier transform to the Lifshitz-Kosevich model [6] is roughly pressure independent. The value of $0.8 \pm 0.1 m_{\mathrm{e}}$ obtained is low for such materials; in other BEDT-TTF salts we observed enhancement from the electronic band mass which was associated with strong correlation in these narrow band conductors [7]. Unfortunately, the Dingle temperature is difficult to measure as there are only few oscillations in the magnetic field attainable, although the rate of increase in amplitude with field does not vary appreciably with pressure.

Under ambient pressure (BEDT-TTF) ${ }_{3} \mathrm{Cl}_{2} 2 \mathrm{H}_{2} \mathrm{O}$ undergoes the transition to an insulating state below about $100 \mathrm{~K}$, which is gradually suppressed by pressure to $6 \mathrm{~K}$ at $10 \mathrm{kbar}$ [4]. According to the recent resistivity measurements, the nature of 


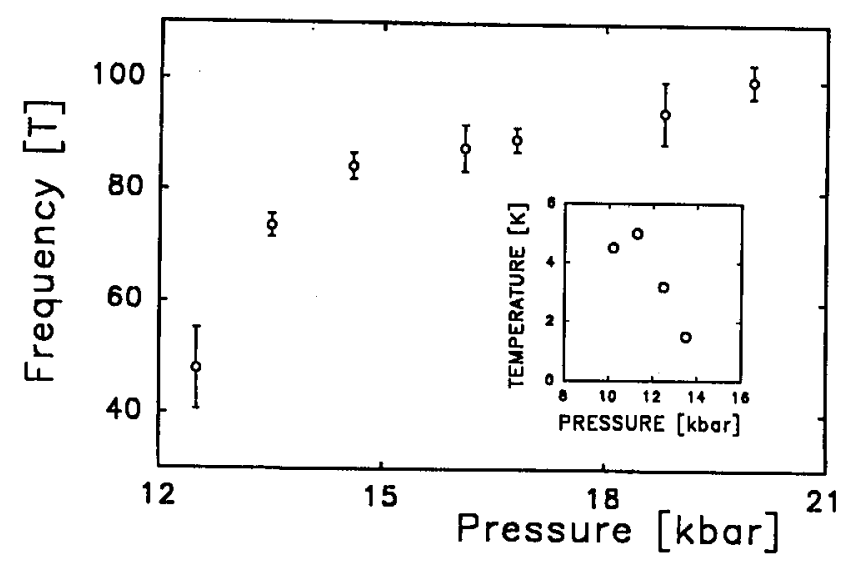

Fig. 2. The measured Shubnikov-de Haas frequency plotted as a function of pressure. The inset is a plot of the observed resistive onset to superconductivity as a function of pressure.

this transition is related to the charge density wave formation caused by nesting of the open sections of the Fermi surface [8]. Pressure also has the effect of forming a closed section of Fermi surface, responsible for the Shubnikov-de Haas oscillations observed above $12.5 \mathrm{kbar}$.

Superconductivity is observed above $0.7 \mathrm{~K}$ in the pressure range $10 \div 14.5 \mathrm{kbar}$ (Fig. 2, inset). The superconducting transition temperature, $T_{\mathrm{c}}$, increases with pressure, peaking at the pressure of $\approx 11.4 \mathrm{kbar}$. With further increase in pressure $T_{\mathrm{c}}$ decreases, until at $\approx 14.5 \mathrm{kbar}$ the onset of superconductivity is not observable at temperatures above $0.7 \mathrm{~K}$. The possible reason for such behaviour is that the structural changes brought about by the application of pressure may lead to changes in the phonon spectrum which affect the formation of the superconducting state.

\section{References}

[1] M.H. Whangbo, J. Ren, B. Kang, J.M. Williams, Mol. Liq. Cryst. 181, 17 (1990).

[2] T. Mori, H. Inokuchi, Chem. Lett., 1657 (1987).

[3] S.D. Obertelli, I.R. Marsden, R.H. Friend, M. Kurmoo, M. Rosseinsky, P. Day, F.L. Pratt, W. Hayes, Springer Proceedings in Physics 51, 181 (1990).

[4] M. Kurmoo, M.J. Rosseinsky, P. Day, P. Auban, W. Kang, D. Jerome, P. Batail, Synth. Met. 27, A425 (1988).

[5] T. Mori, H. Inokuchi, Solid State Commun. 64, 335 (1987).

[6] D. Shoenberg, Magnetic Oscillations in Metals, Cambridge University Press, Cambridge 1984.

[7] J. Caulfield, W. Lubczyński, F.L. Pratt, J. Singleton, D.Y.K. Ko, W. Hayes, M. Kurmoo, P. Day, J. Phys., Condens. Matter 6, 2911 (1994).

[8] S. Demishev, J. Caulfield, J. Singleton, M. Kurmoo, to be published. 